\title{
Exercício físico e gravidez: prescrição, benefícios e contraindicações
}

\author{
Denise Maria Simão Castro' \\ Adriana Magalhães Ribeiro ${ }^{2}$ \\ Larissa de Lima Cordeiro 3 \\ Lorena de Lima Cordeiro 4 \\ Aline Teixeira Alves ${ }^{5}$
}

\section{Resumo}

O objetivo desta revisão bibliográfica foi avaliar a inter-relação do exercício físico com a gravidez, seus benefícios, prescrições e contra-indicações. Foram pesquisados artigos das bases de dados: Scielo, Medline, Lilacs, sendo encontrados 17 artigos. Foram utilizados também como fonte 3 livros. A literatura sugere que a atividade física regular, moderada e controlada, desde o início da gestação, promove benefícios para a saúde materna e fetal com tendência de parecer ser o exercício na água a melhor opção para gestantes.

Palavras-chave: Gravidez. Exercício Físico. Feto. Gestantes.

\section{Introdução}

A mulher durante a gestação sofre várias modificações fisiológicas em seu organismo, tais como: alterações do sistema hormonal, músculo-esquelético, cardiovascular, respiratório, tegumentar, urinário, gastrointestinal e alterações psicológicas (ANJOS; PASSOS; DANTAS, 2003; GOSRKI, 1985; STEPHENSON; O’CONNOR, 2004). Portanto, são necessários cuidados especiais durante esse período para não colocar em risco a mãe e o bebê (SHIMA, 1990).

\footnotetext{
${ }^{1}$ Acadêmica do Centro Universitário de Brasília - UniCEUB, email: loryfisio@yahoo.com.br.

${ }^{2}$ Acadêmica do Centro Universitário de Brasília - UniCEUB, email: loryfisio@yahoo.com.br.

${ }^{3}$ Acadêmica do Centro Universitário de Brasília - UniCEUB, email: loryfisio@yahoo.com.br.

${ }^{4}$ Acadêmica do Centro Universitário de Brasília - UniCEUB, email: loryfisio@yahoo.com.br.

${ }^{5}$ Mestranda em Gerontologia; Docente das disciplinas de Ginecologia e Fisioterapia Aplicada à Ginecologia do UniCEUB, email: aline1304@yahoo.com.br.
} 
É sabido e comprovado que os grandes benefícios trazidos pela atividade física em qualquer fase da vida, trazem melhora em âmbito geral na saúde, nas relações sociais, na qualidade de vida e na prevenção de diversas doenças. Porém, não existe um consenso em relação aos seus reais benefícios, prescrições e contraindicações no período gestacional. O que se sabe é que grande parte da literatura concorda que exercícios nos últimos meses de gravidez devem ser limitados (SHIMA, 1990; BATISTA et al., 2003; CAVALCANTE et al., 2005). Os sistemas locomotor, cardiovascular e respiratório são os que sofrem maior influência durante a prática de atividade física (ANJOS; PASSOS; DANTAS, 2003).

Os exercícios durante a gravidez podem ter riscos associados quando feitos acima do limite materno, em condições desfavoráveis e sem acompanhamento de um profissional capacitado. O que se vê na prática é que os benefícios superam os riscos em potencial, se for tomado o devido cuidado ao ministrar o programa de exercícios (SOUZA et al., 2000).

O presente trabalho tem por objetivo apresentar, por meio de revisão de literatura, considerações levantadas a respeito da interação exercício-gravidez, seus benefícios para a mãe e o bebê, suas contraindicações e sua prescrição.

\section{Metodologia}

Para a elaboração deste trabalho de revisão, foram utilizados artigos publicados nos últimos 11 anos, pesquisados na base de dados da Bireme, por meio dos serviços da Medline, Scielo e Lilacs. As publicações foram selecionadas mediante busca com os seguintes descritores: exercícios, água e gravidez; atividade física e gravidez; exercícios e gestação; hidroterapia e gravidez; educação física e gravidez; hidroterapia e gestação; pregnancy e exercise; exercise e low back pain. Foram selecionados 17 artigos nas línguas portuguesa e inglesa e utilizados também como fonte três livros. Os critérios para escolha dos artigos e livros envolveram todo tipo de estudo que se baseasse no tema escolhido, não havendo exclusões. 


\section{Discussão}

A atividade física é uma movimentação corporal que despende um gasto energético muito maior que no estado de repouso, ocasionado pelas mudanças na composição corporal. A melhoria da força física, flexibilidade e resistência proporcionadas pelo exercício podem ser de grande valia para mulheres no período gestacional. Antigamente as mulheres não tinham o acompanhamento necessário durante o período gestacional, dificultando a realização da atividade física. Com o tempo, a importância de um pré-natal durante toda a gravidez foi sendo reconhecida, possibilitando uma melhor assistência à mãe e ao bebê, tornando-se possível a realização do exercício físico na gestação sem complicações (BATISTA et al., 2003).

O exercício pré-natal tem como objetivo principal a melhoria da qualidade de vida da gestante por meio da aplicação de um programa de exercícios e de orientações sobre a realização das atividades cotidianas, as quais necessitam ser adaptadas às circunstâncias da gestação, sendo importante também um apoio psicológico (ANJOS; PASSOS; DANTAS, 2003).

Além da melhoria da qualidade de vida, diversos outros benefícios são proporcionados pela atividade física durante a gravidez, tais como, a melhoria da qualidade do sono, da postura da grávida, da diástase do músculo reto-abdominal e do desenvolvimento de varicosidades (DERTKIGIL et al., 2005).

Um estudo de revisão mostrou vários benefícios da prática da atividade física na gestação como: prevenção e redução de lombalgias, de dores das mãos e pés e de estresse cardiovascular; fortalecimento da musculatura pélvica; redução de partos prematuros e cesáreas e das dores no parto; maior flexibilidade e tolerância à dor; controle do ganho ponderal e elevação da autoestima da gestante. No feto, foram observados aumento do peso ao nascer e melhoria da condição nutricional. Caso ocorra prematuridade, esta se deve à atividade de intensidade excessiva tanto física quanto ocupacional (SHIMA, 1990; BATISTA et al., 2003).

É importante observar que a atividade física na gestação não afeta significantemente o peso fetal, mas mostra uma tendência a um menor peso em fetos de gestantes a termo e que se mantiveram ativas durante todo o terceiro trimestre da gravidez (SHIMA, 1990; DERTKIGIL et al., 2005). 
94 Universitas: Ciências da Saúde, Brasília, v. 7, n. 1, p. 91-101, 2009

Denise Maria S. Castro, Adriana M. Ribeiro, Larissa L. Cordeiro,

Lorena L. Cordeiro, Aline T. Alves

Algumas recomendações são necessárias para a prática de exercício nesse período da vida da mulher. Primeiramente, é importante uma prescrição médica, devendo ela especificar as atividades não recomendadas à gestante e a intensidade ideal para o trabalho. Outro ponto importante, segundo os autores, é que não se deve objetivar o aumento do condicionamento físico, pois com a gestante ocorre exatamente o inverso: sua resistência inicial tende a diminuir. Deve-se, também, evitar o aumento na temperatura corporal e a perda hídrica. As gestantes que praticavam atividade física antes de engravidar não devem iniciá-las antes de duas semanas após a concepção, podendo variar de acordo com as respostas fisiológicas individuais apresentadas (CHISTÓFALO; MARTINS; TUMELERO, 2003).

É muito comum a afirmação de que para se ter uma boa saúde deve-se praticar exercício físico, porém não existe um consenso em relação ao tipo, intensidade, época e duração dele no período gestacional (CAVALCANTE et al., 2005). Segundo Shima (1990), ainda são controversos e indefinidos os seus reais benefícios, prescrições e contraindicações nesse período. O que se sabe é que a maioria das publicações concorda que exercícios nos últimos meses de gravidez devem ser limitados.

Em relação, porém, à atividade aeróbica, já há evidências na literatura sobre os seus benefícios durante a gestação, trazendo benefícios respiratórios, auxiliando no retorno venoso, melhorando as trocas gasosas e a capacidade aeróbica, exigindo um menor esforço para a gestante, evitando assim complicações (CAVALCANTE et al., 2005). Os exercícios aeróbicos auxiliam também no controle do peso, na manutenção do condicionamento da mãe e na redução do risco de diabetes gestacional por meio de uma melhor utilização da glicose e do aumento da sensibilidade à insulina (LIMA; OLIVEIRA, 2005). Segundo Chistófalo, Martins e Tumelero (2003), as prescrições iniciais para esse tipo de exercício incluem: frequência de 3 vezes semanais, em dias intercalados, com duração de 30 a 45 minutos sendo que a frequência cardíaca média deve se manter entre 130 e 150 batimentos por minuto.

Quanto aos exercícios físicos resistidos, quando realizados em intensidade adequada para o período gestacional, promovem melhora na resistência e flexibilidade muscular, sem aumento no risco de lesões, complicações relativas à gestação ou ao peso do feto ao nascer. Também são benéficos os exercícios para a musculatura pélvica, prevenindo a incontinência urinária associada à gravidez (LIMA; OLIVEIRA, 2005). 
Há um consenso na literatura de que exercícios de intensidade moderada são os adequados para o período gestacional. Durante uma gravidez não complicada, eles promovem melhora na resistência e flexibilidade muscular, sem aumento nos riscos de lesões e complicações. Além disso, os exercícios físicos mantêm a saúde mental e emocional da mulher grávida, protegendo-a, inclusive, da depressão puerperal (ACOG, 2002; LIMA; OLIVEIRA, 2005; BARACHO, 2007).

Outras recomendações para os exercícios durante a gravidez são: evitar treinos em que a frequência cardíaca exceda $140 \mathrm{bpm}$; realizar os exercícios em número de 3 a 4 vezes na semana, durante 20 a 30 minutos; evitar contrações isométricas máximas, assim como exercícios na posição supina e em ambientes quentes e piscinas muito aquecidas (LIMA; OLIVEIRA, 2005; MENEGHELO; ANDRADE, 2000; PREVEDEL et al., 2003).

Os exercícios realizados na água vêm se destacando como ideais para o período gestacional. A natação é a mais recomendada para esse período devido à propriedade da flutuabilidade. Sendo a atividade feita na água, os joelhos recebem menor sobrecarga, há redução de edema pela ação da pressão hidrostática, que facilita e estimula a passagem de líquido do meio intersticial para o intravascular; ocorre também maior gasto energético, aumento da capacidade cardiovascular e o relaxamento corporal, reduzindo assim, desconfortos músculo-esqueléticos, comuns nesse período. Além desses, foram relatados também que exercícios sob imersão diminuem as contrações uterinas e propiciam maior conforto à mulher, principalmente no último semestre, devido ao grande volume abdominal (BATISTA et al., 2003; DERTKIGIL et al., 2005; MACIEL et al., 2003; PREVEDEL et al., 2003; SACHELLI, ACCACIO, RADL, 2007).

Outros benefícios da atividade física em imersão são mostrados por Prevedel et al. (2003), em estudo prospectivo, de coorte, aleatorizado. Os autores apontam o incremento da diurese e o controle do estresse. A diurese, estimulada pela imersão, pode ter efeitos positivos sobre a gestante portadora de retenção hídrica e, portanto, de interesse para as gestações complicadas por síndromes hipertensivas (PREVEDEL; CALDERON; RUDGE, 2003).

Em estudo prospectivo, de coorte, com 41 mulheres de gestação de baixo risco, divididas em um grupo controle (não praticantes da hidroterapia) e um grupo 
96 Universitas: Ciências da Saúde, Brasília, v. 7, n. 1, p. 91-101, 2009

Denise Maria S. Castro, Adriana M. Ribeiro, Larissa L. Cordeiro,

Lorena L. Cordeiro, Aline T. Alves

estudo (praticantes de hidroterapia), mostrou que, durante a gravidez, o ganho de peso materno não se modificou em função da hidroterapia. Neste estudo, também chegou-se à conclusão que a hidroterapia não determinou prejuízo aos recém-nascidos das gestantes estudadas que exerciam essa prática (PREVEDEL et al., 2003).

Outros benefícios ocasionados pelo simples fato de estar dentro da água são as lombalgias, que podem ser aliviadas pelo exercício aquático, ,pois permite que a ação da gravidade atue de forma menos intensa. Em decorrência, o peso corporal é aliviado e melhor suportado; a postura é corrigida durante a hidroterapia, reduzindo a sensação do desconforto gestacional (PREVEDEL et al., 2003). Waller, Lambeck e Daly (2009) também concluem, por meio de revisão sistemática, que o exercício aquático terapêutico traz benefícios potenciais a pacientes gestantes que sofrem de lombalgia. Esse fato é de grande relevância visto que grande parte das mulheres grávidas tem algum tipo de desconforto músculo-esquelético durante a gestação, entre os quais um dos principais é a lombalgia. Esse sintoma afeta cerca de 50\% das gestantes em nível de população mundial (LIMA; OLIVEIRA, 2005).

Lima e Oliveira (2005) afirmam que um programa de exercícios executado três vezes por semana durante a segunda metade da gravidez parece reduzir a intensidade das dores lombares, aumentando também a flexibilidade da coluna.

Cada sessão na piscina tem uma duração de 45 a 60 minutos, não devendo ultrapassar uma hora, evitando-se assim a fadiga da grávida. O nível ideal da água para a realização dos exercícios é na altura do esterno, cobrindo o abdômen sem colocar pressão sobre as mamas facilitando a manutenção da postura vertical. A utilização de alguns materiais como pranchas de natação, bolas, halteres próprios para a água, bastões e flutuadores de várias formas tornam a sessão mais prazerosa para a gestante (ANJOS; PASSOS; DANTAS, 2003). Para que o exercício aquático seja benéfico, é necessário que a temperatura da água não seja muito quente, devendo, portanto, estar entre $28^{\circ} \mathrm{C}$ e $30^{\circ} \mathrm{C}$ (BATISTA et al., 2003).

A fisioterapia, por meio da cinesioterapia, também traz muitos benefícios às gestantes por meio da proposta de adaptações osteoarticulares da mãe. É uma técnica que deveria ser incluída em programas de assistência multidisciplinar por serem práticas, de baixo custo e proporcionarem significativa melhoria na qualidade de vida nesse período da mulher (CONTI et al., 2003). 
A gestante deve ser encaminhada ao fisioterapeuta e passar por uma avaliação completa antes do início das sessões. No exame postural, o fisioterapeuta poderá observar a gestante em várias posturas durante a realização de alguns movimentos e também ao caminhar, detectando assim as principais dificuldades da gestante para então elaborar uma conduta fisioterápica (ANJOS; PASSOS; DANTAS, 2003).

Devemos, porém, salientar que alguns tipos de exercícios, como os que exigem equilíbrio preciso, os de atividade competitiva com movimentos repentinos e saltos, artes marciais, levantamento de peso, flexão ou extensão profunda (devido à frouxidão ligamentar já existente na gravidez) e mergulho, são contraindicados durante o período gestacional. Jogos com bolas ou que possam causar traumas abdominais, atividades aeróbicas em alta altitude e exercícios na posição supino após o terceiro trimestre (pois podem obstruir o retorno venoso) não são recomendados (BATISTA et al., 2003; DERTKIGIL et al., 2005; WANG; APGAR, 1998).

As mulheres grávidas que não possuem contraindicações devem ser incentivadas a realizar exercícios aeróbicos, de resistência muscular e alongamento. As atividades escolhidas não devem apresentar risco de perda de equilíbrio e traumas. Esportes de contato, com alto risco de colisão não são indicados (DAVIES et al., 2003; LIMA; OLIVEIRA, 2005).

A realização de qualquer exercício deve ser muito bem supervisionada nas gestantes, principalmente nos últimos meses de gravidez. Os profissionais devem estar atentos aos sinais e sintomas de perigo apresentados durante a prática, devendo ser interrompida imediatamente caso eles apareçam. Alguns sinais claros para interrupção do exercício são: perda de líquido amniótico, dor no peito, sangramento anormal, dispneia, dor abdominal, contrações uterinas, redução dos movimentos fetais, náuseas, desconfortos. Todos os sintomas devem ser analisados, podendo alguns deles serem resolvidos apenas com a mudança do tipo, intensidade ou duração do exercício. Porém, algumas mulheres portadoras de algumas patologias como doenças cardíacas descompensadas, doença pulmonar restritiva, placenta prévia, pré-eclampsia, cérvix incompletas ou multíparas com riscos de pré-maturidade têm contraindicação absoluta durante o período gestacional (BATISTA et al., 2003). 
As grávidas que apresentam restrições relativas como anemia, doenças tireoidianas, diabetes mellitos descompensado, obesidade mórbida e sedentarismo, ao serem liberadas para a atividade, devem ser muito bem monitoradas (LIMA; OLIVEIRA, 2005).

Dessa forma, verificamos que os exercícios durante a gravidez podem ter riscos associados quando feitos acima do limite materno, em condições desfavoráveis e sem acompanhamento de um profissional capacitado. Porém, o que se vê na prática é que os benefícios superam os riscos em potencial, se for tomado o devido cuidado ao ministrar o programa de exercícios (SOUZA et al., 2000). Lima e Oliveira (2005) não especificaram qual atividade física é a mais indicada durante a gravidez, mas destacaram sua importância nesse período, desde que todos os cuidados sejam tomados.

\section{Conclusão}

A atividade física durante a gravidez traz inúmeros benefícios para a mãe e para o feto, desde que sejam tomados os devidos cuidados quanto ao tipo, duração e intensidade dos exercícios, respeitando as contraindicações e patologias associadas com acompanhamento profissional e indicação médica de maneira individualizada.

Podem ser realizados exercícios aeróbicos, de alongamento e que trabalham resistência muscular, de intensidade moderada e duração de aproximadamente 3 vezes por semana, com frequência cardíaca máxima em torno de $140 \mathrm{bpm}$. Já exercícios de alta intensidade são contraindicados por trazerem riscos à saúde da mãe e do bebê.

Os benefícios decorrentes do exercício físico nesse período da vida da mulher são inúmeros e estão relacionados com a melhoria de diversas alterações que ocorrem nas grávidas. Essas alterações estão presentes nos sistemas musculoesquelético, cardiovascular, respiratório, entre outros. Porém, existem contraindicações que são: aqueles que exigem equilíbrio preciso, os competitivos, com movimentos repentinos e saltos, artes marciais, levantamento de peso, flexão ou extensão profunda, mergulho, aqueles que podem causar traumas abdominais, atividades aeróbicas em alta altitude e exercícios na posição supino após o terceiro trimestre. 
Além disso, algumas patologias também têm contraindicação absoluta para exercício físico nesse período. São elas: doenças cardíacas descompensadas, doença pulmonar restritiva, placenta prévia, pré-eclampsia, cérvix incompleto ou multíparas com riscos de pré-maturidade.

A hidroterapia e os exercícios realizados na água são os mais recomendados pela literatura. A água possui propriedades físicas peculiares que a transformam em um meio terapêutico muito eficiente e seguro para a realização da sessão. $\mathrm{O}$ relaxamento, a menor sobrecarga articular, a redução do edema e o maior conforto proporcionado à mulher são alguns dos benefícios trazidos por esse tipo de exercício. Porém, é necessário que a água não seja muito quente, estando em temperatura aproximada de $28^{\circ} \mathrm{C}$.

Os músculos respiratórios precisarão trabalhar contra a soma das pressões hidrostática e intra-abdominal, melhorando assim o condicionamento e tônus dessa musculatura e a capacidade inspiratória da gestante.

A prática de exercício físico deve ser encorajada pelos profissionais de saúde e realizada conforme a motivação da gestante. Não existindo nenhuma padronização quanto ao tipo de exercício, a grávida pode optar pelo que mais lhe agrada desde que tenha acompanhamento médico e as contraindicações sejam respeitadas.

\section{Physical exercise and pregnancy: prescription, benefits and restrictions}

\section{Abstract}

The objective of this bibliographical revision was to evaluate the interrelation of the physical exercise and the pregnancy, your benefits, prescriptions and restrictions. The researches were taken from the databases Scielo, Medline, Lilacs, being found 17 papers. Three books were either utilized. The literature suggests that, the regulated, moderate and controlled physical activity since the beginning of the gestation promotes benefits for the maternal and fetal health with tendency of seeming to be the exercise in the water the best option for pregnant women.

Keywords: Pregnancy. Physical Exercise. Fetus. Pregnant. 
Universitas: Ciências da Saúde, Brasília, v. 7, n. 1, p. 91-101, 2009
Denise Maria S. Castro, Adriana M. Ribeiro, Larissa L. Cordeiro,

Lorena L. Cordeiro, Aline T. Alves

\section{Referências}

ACOG. Exercise during pregnancy and the postpartum period. International Journal of Gynecology \& Obstetrics, [S.1.], n. 77, p. 79-81, 2002.

ANJOS, G. C. M.; PASSOS, V.; DANTAS, A. R. Fisioterapia aplicada á fase gestacional: uma revisão da literatura. Trabalho de Conclusão de Curso (Graduação em Fisioterapia)- Universidade Federal de Pernambuco, Pernambuco, 2003.

BARACHO, E. Fisioterapia aplicada à obstetrícia, uroginecologia e aspectos de mastologia. 4. ed. Rio de Janeiro: Guanabara-Koogan, 2007.

BATISTA. D. C. et al. Atividade física e gestação: saúde da gestante não atleta e crescimento fetal. Revista Brasileira de Saúde Materno Infantil, Recife, v. 3, n. 2, p. 151-158, abr./jun. 2003.

CAVALCANTE, S. R. et al. Exercícios físicos na água durante a gestação. Femina, Rio de Janeiro, v. 33, n. 7, p. 547-551, jul. 2005.

CHISTÓFALO, C.; MARTINS, A. J.; TUMELERO, S. A prática de exercício físico durante o período de gravidez. Revista Digital, Porto Alegre, n. 59, abr. 2003.

CONTI, M. H. S. et al. Efeito de técnicas fisioterápicas sobre os desconfortos músculo-esqueléticos da gestação. Revista Brasileira de Ginecologia e Obstetrícia, Rio de Janeiro, v. 25, n. 9, p. 647-653, 2003.

DAVIES, G. A. L. et al. Exercise in pregnancy and the postpartum period. Joint SOGC/CSEP Clinical Practice Guideline, [S.1.], n. 129, june 2003. Disponível em: <http://www.sogc.org/guidelines/public/129E-JCPG-June 2003.pdf>. Acesso em: 17 maio 2009.

DERTKIGIL, M. S. J. et al. Líquido amniótico, atividade física e imersão em água na gestação. Revista Brasileira de Saúde Materno Infantil, [S.l.], v. 5, n. 4, p. 403410, out./dez. 2005.

LIMA, F. R.; OLIVEIRA, N. Gravidez e exercício. Revista Brasileira de Reumatologia, [S.1.], v. 45, n. 3, p. 188-190, maio/jun. 2005.

MACIEL, F. A. et al. Exercício físico na gavidez. Femina, Rio de Janeiro, v. 31, n. 3, p. 253-257, abr. 2003.

MENEGHELO, R. S.; ANDRADE, J. Atividade física na gravidez: riscos e benefícios. In: ANDRADE, J. Patologias cardíacas da gestação. São Paulo: Edusp, 2000. (Acadêmica, 33). 
PREVEDEL T. T. S. et al. Repercussões maternas e perinatais da hidroterapia na gravidez. Revista Brasileira de Ginecologia e Obstetrícia, Rio de Janeiro, v. 25, n. 1, p. 53-59, 2003.

PREVEDEL T. T. S; CALDERON, I. M. P.; RUDGE, M. V. C. A prática da atividade física na gestação: aspectos atuais. Femina, Rio de Janeiro, v. 31, n. 9, p. 749-753, out. 2003.

SACHELLI, T.; ACCACIO, L. M. P.; RADL, A. L. M. Fisioterapia aquática. Barueri: Manole, 2007.

SHIMA, H. Considerações sobre a atividade física na gestação. Revista da Escola de Enfermagem da USP, São Paulo, v. 24, n. 3, p. 389-396, dez. 1990.

SOUZA, E. L. B. L. Fisioterapia aplicada à obstetrícia e aspectos de neonatologia: uma revisão multidisciplinar. 2. ed. Belo Horizonte: Health, 2000.

STERPHESON, R. G.; O'CONNOR, L. J. Fisioterapia aplicada à ginecologia e obstetrícia. 2 ed. Barueri: Manole, 2004.

WALLER, B.; LAMBECK, J.; DALY, D. Therapeutic aquatic exercise in the treatment of low back pain: a systematic review. Clinical Rehabilitation, [S.l.], v. 23, p. 3-14, 2009.

WANG, T. W.; APGAR, B. S. Exercise during pregnancy. American Family Physician, [S.1.], v. 57, p. 1846-1852, 1998. 
Para publicar na revista Universitas Ciências da Saúde, entre no endereço eletrônico www.publicacoesacademicas.uniceub.br. Observe as normas de publicação, facilitando e agilizando o trabalho de edição. 